\title{
PEDIATRICS
}

\section{An oligosaccharide can prevent necrotizing enterocolitis in rats}

Disialyllacto- $N$-tetraose, an oligosaccharide found in human milk, protects against necrotizing enterocolitis in a rat model of this common and often fatal illness.

"Human milk oligosaccharides are complex glycans that are highly abundant in human milk, but not in infant formula," explains corresponding author Lars Bode (University of California, San Diego, USA). "Intriguingly, breast-fed infants are at a much lower risk of developing necrotizing enterocolitis than formulafed infants." These observations led the researchers to investigate whether the oligosaccharides in human milk could protect against necrotizing enterocolitis. As such a study would be difficult in humans (as it is not yet possible to synthesize large quantities of the oligosaccharides), the researchers used a rat model of necrotizing enterocolitis.

The researchers isolated human oligosaccharides from donated breast milk, which was then added to formula milk. Rat pups were fed formula that either did or did not contain the human milk oligosaccharides and were exposed to hypoxia events. Ileum sections were taken to determine whether signs of necrotizing enterocolitis were present, and 2D chromatography was used to identify which oligosaccharide was the most effective.

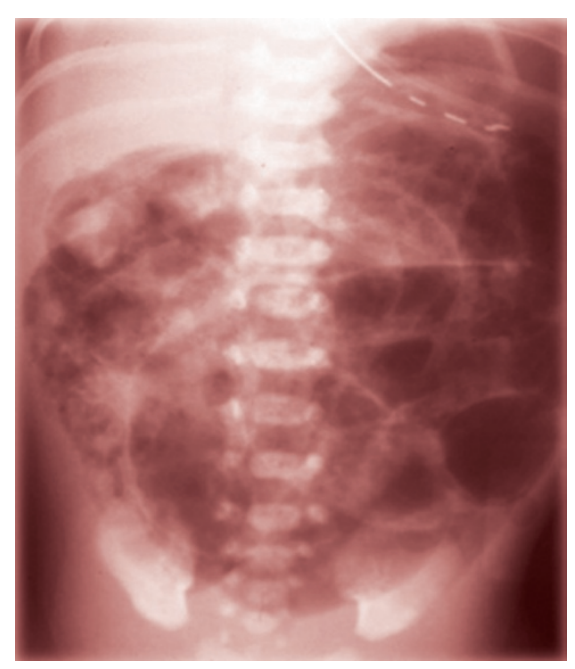

An X-ray from a human preterm infant with necrotizing enterocolitis, showing typical pneumatosis intestinalis. Courtesy of F. Mannino.

4-day survival was $95 \%$ for the rats fed formula with added oligosaccharides, compared with $73 \%$ for those just fed formula and $100 \%$ of the controls that were fed rat milk. Interestingly, just $77 \%$ of rats fed formula with galacto-oligosaccharides (which are supposed to mimic human milk oligosaccharides) survived at 4 days. Histological examination of the ilea of the rats confirmed that human milk oligosaccharides reduced the signs of necrotizing enterocolitis.
As human milk contains over 150 different oligosaccharides, it was important to determine which one was responsible for the protective effect. A 2D chromatography analysis revealed that disialyllacto- $N$-tetraose was the key component.

The authors are now working on identifying the mechanisms that underlie this protective effect, which could improve our understanding of the etiology of necrotizing entercolitis. Bode notes that this research could lead to the oligosaccharide composition of a mother's milk being used as a noninvasive biomarker to determine her preterm infant's risk of developing necrotizing enterocolitis. "We are now looking to develop a strategy to synthesize disialyllacto- $N$-tetraose and then test it in a human intervention study," concludes Bode. "In the end, we encourage mothers to breastfeed their infants to provide them with the very best protection against necrotizing enterocolitis."

\section{Claire Greenhill}

Original article Jantscher-Krenn, E. et al. The human milk oligosaccharide disialyllacto-N-tetraose prevents necrotising enterocolitis in neonatal rats. Gut doi:10.1136/ gutjnl-2011-40404 